\title{
Have patient pathways for penetrating chest injuries improved since designation as a London major trauma centre?
}

\author{
JR Pallett ${ }^{*}$, J Olding, T Hurst, M Tunnicliff, JW Keep \\ From London Trauma Conference 2012 \\ London, UK. 4-7 December 2012
}

\section{Background}

King's College Hospital NHS Foundation Trust was designated a Major Trauma Centre for the South East London Trauma Network in April 2010. Penetrating trauma accounts for a high proportion of the Trauma Team caseload at this centre and various initiatives have been made to improve the quality of the major penetrating trauma pathways.

\section{Methods}

A retrospective cohort study of penetrating chest trauma cases seen by the Trauma Team from April 2010 and April 2011 was compared. Statistical analysis of unpaired means was calculated with the $z$-test and twotailed p-values.

\section{Results}

25 cases from April, May and June 2010 (100\% male, mean age 28.6 years) and 28 cases from April, May and June 2011 ( $100 \%$ male, mean age 24.1 years) were indentified. In 2010, mean time to CXR was 18.86 minutes, $95 \%$ CI $(12.97,24.75)$ and mean time to CT was 93.10 minutes $(61.27,124.93)$. In $40 \%$ of cases use of $e F A S T$ was documented. In total, $91.3 \%$ underwent CT scanning of the thorax. In February 2011, a Standard Operating Procedure (SOP) was introduced along with pre-registration of patients prior to arrival, use of a designated trauma CT scanner and increased training of $e F A S T$. In 2011, mean time to CXR was 10.45 minutes, $95 \%$ CI $(7.55,13.35)$ and mean time to CT was 46.94 minutes, $95 \%$ CI $(26.36,67.52)$. In $54 \%$ of cases use of $e F A S T$ was documented. In total, $60.7 \%$ of cases underwent CT scanning of the thorax. Comparing this data over time, both time to CXR ( $\mathrm{p}=0.012)$ and time to CT $(\mathrm{p}=0.000024)$ have significantly decreased along with increased utilisation of $e F A S T$ and reduced use of CT scanning in accordance with the SOP.

\section{Conclusion}

In the intervening year as a Major Trauma Centre, there is quite strong evidence $(\mathrm{p}<0.05)$ for CXR and very strong evidence $(\mathrm{p}<0.0001)$ for $\mathrm{CT}$, that time to diagnostic imaging has significantly improved. The introduction of an SOP incorporating physiological parameters and zone of chest injury along with increased use of $e F A S T$ has contributed to a reduction in the use of inappropriate CT scanning in accordance with recent evidence-based guidance [1].

\section{Published: 28 May 2013}

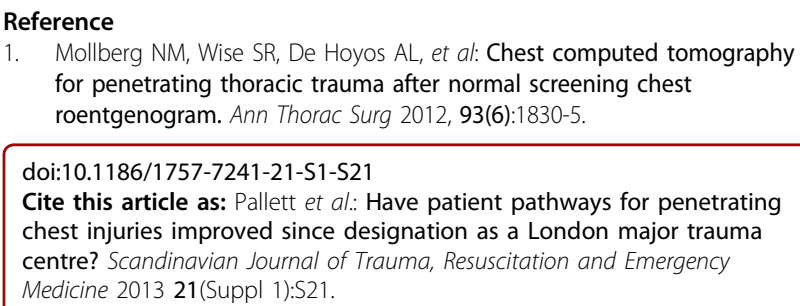

Cite this article as: Pallett et al:: Have patient pathways for penetrating chest injuries improved since designation as a London major trauma centre? Scandinavian Journal of Trauma, Resuscitation and Emergency Medicine 2013 21(Suppl 1):S21. 